Copyright (C) 2006 IEEE. Reprinted from

V. V. S. Nagesh Polu, Bruce G. Colpitts and Brent R. Petersen, "Symbol-wavelength MMSE gain in a multi-antenna UWB system," Proceedings of the $4^{\text {th }}$ Annual Communication Networks and Services Research Conference (CNSR 2006), vol. 1, (Moncton, NB, Canada), pp. 95-99, May 24-25, 2006.

This material is posted here with permission of the IEEE. Internal or personal use of this material is permitted. However, permission to reprint/republish this material for advertising or promotional purposes or for creating new collective works for resale or redistribution must be obtained from the IEEE by sending a blank email message to pubs-permissions@ieee.org.

By choosing to view this document, you agree to all provisions of the copyright laws protecting it.

CNSR 2006 was technically co-sponsored by the IEEE Communications Society and the ACM.

This paper received the Conference Best Paper Award. 
Proceedings of $4^{\text {th }}$ Annual Communication Networks and Services Research Conference (CNSR 2006),

(Moncton, NB, Canada), May 24-25, 2006.

\title{
Symbol-wavelength MMSE gain in a multi-antenna UWB system
}

\author{
V. V. S. Nagesh Polu, Bruce G. Colpitts, and Brent R. Petersen \\ University of New Brunswick \\ Department of Electrical and Computer Engineering \\ Fredericton, NB, Canada - E3B 5A3 \\ Email: nagesh.polu@ieee.org, colpitts@unb.ca, b.petersen@ieee.org
}

\begin{abstract}
Ultra wideband $(U W B)$ technology has the potential to provide high speed data services. These speeds can be greatly increased by using multiple input multiple output (MIMO) techniques. Multiple antennas are used to achieve spatial diversity and these antenna elements must be separated properly to have uncorrelated communication signals. The separation can be on the scale of a symbol wavelength ([speed of light]/[symbol rate]). We have designed a printed circular disc monopole and measured the UWB radio channel. Finally, we used the measurements to show the minimum mean squared error (MMSE) performance gain which occurs when the receiver antennas are separated on the scale of a symbol wavelength.
\end{abstract}

Keywords: MIMO systems, adaptive equalizers, antenna measurements

\section{Introduction}

UWB technology seems to be an efficient solution for the ultra high-speed data services (i.e. speeds up to 480 Mbps) in wireless personal area network (WPAN) environments (i.e. in ranges of $\approx 10 \mathrm{~m}$ ). This is because of its low cost and very simple architecture. Recently the Federal Communication Commission (FCC) allocated a very wide spectrum of $7.5 \mathrm{GHz}$ (i.e. from $3.1 \mathrm{GHz}$ to $10.6 \mathrm{GHz}$ ) for UWB applications. UWB technology uses two different modulation techniques to cover entire UWB spectrum, one technique is Impulse-UWB, which uses a very short duration pulses to transmit data over a large bandwidth and the other is a multicarrier UWB technique [1]. The FCC defines UWB as a signal with either a fractional bandwidth of 20 percent of the center frequency or a minimum of $500 \mathrm{MHz}$ [1].

Important recent work shows the dependence of correlated interference on antenna element spacing [2].
The authors defined a parameter called chiplength ([speed of light]/[chip rate]), which can be used to separate antennas in a multi antenna system to have uncorrelated interference at the antenna branches. Similar work describes the separation of the receiver antennas on the scale of a signalling wavelength to achieve spatial multiplexing [3]. Chamchoy et al. showed that there is a high degree of correlation between the channels when the antennas are separated based on half the carrier wavelength [4]. In this paper we investigated the MMSE performance of an UWB system in a line of sight $(\mathrm{LoS})$ scenario, when the receiver antennas are separated on the scale of a symbol wavelength $\left(\lambda_{T}\right)$ to have uncorrelated communication signals at the receiver. The uncorrelation is showed in terms of MMSE gain with increasing separation.

This paper is organized as follows: section 2 describes the system model, section 3 is about the designed antenna for UWB applications, section 4 is about UWB channel measurements, section 5 contains the simulation results of the MMSE equalizer, followed by conclusions and references [5].

\section{Two-by-Two System and Channel Model}

The two-by-two system and channel model will be discussed in this section.

\subsection{Channel Model}

The two-by-two baseband channel model consists of four baseband impulse channels between two users and two receive antennas and it is given by

$$
\mathbf{h}(t)=\left(\begin{array}{ll}
h_{11}(t) & h_{12}(t) \\
h_{21}(t) & h_{22}(t)
\end{array}\right)
$$


and its Fourier transform representation is

$$
\mathbf{H}(f)=\left(\begin{array}{ll}
H_{11}(f) & H_{12}(f) \\
H_{21}(f) & H_{22}(f)
\end{array}\right) .
$$

\subsection{System Model}

The two-by-two system model is shown in the Figure 1. In this paper we consider the uplink of the UWB system with two receive antennas and two simultaneous users [6]. The data is a zero-mean random process and is passed through a pulse shaping filter for transmission into the channel. The signal transmitted from user $j$ is of the form

$$
s_{j}(t)=\sum_{n=-\infty}^{\infty} d_{j}(n) p(t-n T)
$$

where $j$ represents the user number, either 1 or $2, d_{j}(n)$ is binary data which takes values of $\{-1,1\}$ and the pulse shaping filter $p(t)$ is a raised cosine type with $100 \%$ excess bandwidth. Finally the data signal from the users is transmitted through a baseband channel, whose impulse response is given by $\mathbf{h}(t)$ and its equivalent frequency domain representation is $\mathbf{H}(f)$. The

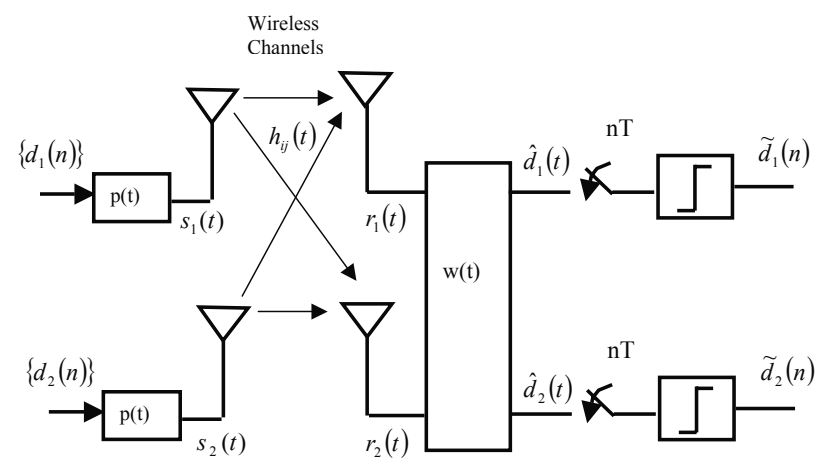

Figure 1. Two-by-Two system and channel model

signal at the receiving antenna is the convolution of the transmitted signal and the baseband channel impulse response plus noise and is given by

$$
\mathbf{r}(t)=\mathbf{h}(t) \otimes \mathbf{s}(t)+\eta(t)
$$

where $\otimes$ denotes matrix convolution and $\eta(t)$ is a zeromean additive white Gaussian noise. The received signal at the antenna is passed through a receiving filter whose impulse response is $\mathbf{w}(t)$. The output of the receiving filter is the convolution of $\mathbf{r}(t)$ and $\mathbf{w}(t)$ (i.e. $\mathbf{w}(t) \otimes \mathbf{r}(t))$. The receiving filter coefficients are updated based on the MMSE criterion determined during training. The received signal $\mathbf{r}(t)$ and the receiving filter $\mathbf{w}(t)$ are given by

$$
\mathbf{r}(t)=\left(\begin{array}{l}
r_{1}(t) \\
r_{2}(t)
\end{array}\right)
$$

$$
\mathbf{w}(t)=\left(\begin{array}{ll}
w_{11}(t) & w_{12}(t) \\
w_{21}(t) & w_{22}(t)
\end{array}\right)
$$

\section{Antenna Design}

In order to make UWB channel measurements we need antennas that have a very wide impedance bandwidth and near omni-directional characteristics for the entire UWB spectrum. We searched for small UWB antennas which have the potential for use in UWB devices $[7,8]$. Finally we simulated and fabricated the printed circular disc monopole antenna specified by Liang et al. [7]. The parameters specified are modified slightly because we used a substrate with a different thickness. The length and width of the designed antenna is $50 \mathrm{~mm}$ and $42 \mathrm{~mm}$ respectively. The antenna simulations were performed using CST Microwave Studio and antennas were fabricated on an FR-4 substrate whose dielectric constant is 4.2 . A picture of the fabricated antenna is shown in Figure 4 . The antennas were measured using an HP 8510B Vector Network Analyzer (VNA) and the SWR plot of the measured and simulated curves is shown in Figure 2. It is clear from Figure 2 that the designed antenna has an SWR of 2.2:1 or better in the specified band.

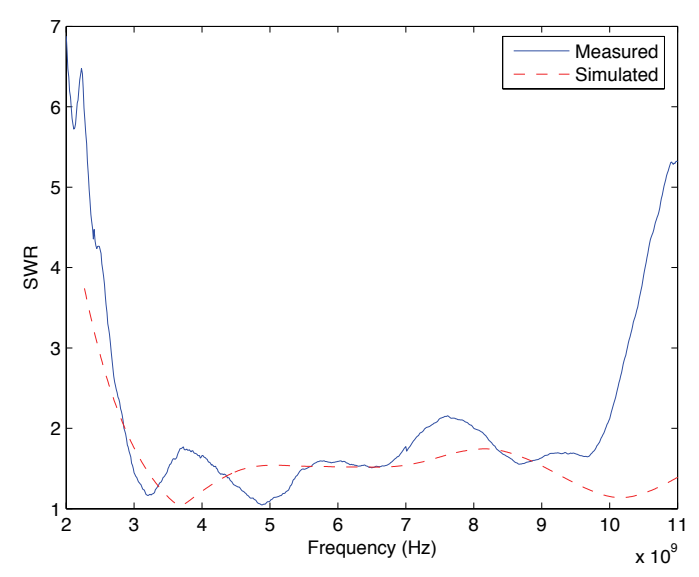

Figure 2. Comparison of measured and simulated $S W R$ 


\section{UWB Channel Measurement}

The channel measurement procedure and the experimental setup used to measure UWB channels will be discussed in this section.

\subsection{Experimental Setup}

The transmitting and receiving antennas are connected to the two ports of the VNA using coaxial cables of length about $2 \mathrm{~m}$ and the system is calibrated including the cables, so the losses in the cables can be neglected and the response obtained on the VNA is the combined UWB channel and antenna response. The transmit ( $\mathrm{Tx})$ and receive $(\mathrm{Rx})$ antennas are in an LoS scenario and kept at a height of $120 \mathrm{~cm}$ from the floor and the distance between Tx and Rx antennas is approximately $60 \mathrm{~cm}$, but varies as receiver antenna element $2\left(R x_{2}\right)$ is moved. The antenna elements at the receiver are separated on the scale of a symbol wavelength as shown in Figure 3.

\subsection{Channel Measurement Procedure}

UWB channel measurements are performed using frequency domain measurement technique [9]. The channel's complex frequency response data from the HP 8510B VNA is collected using a computer running LabVIEW ${ }^{\circledR}$ software which is connected using a GPIB interface. These measurements were performed in a research laboratory environment. Frequency domain measurements are obtained by sweeping the VNA from $3 \mathrm{GHz}$ to $10 \mathrm{GHz}$ in 801 points with a frequency step of $8.75 \mathrm{MHz}$ and the time domain impulse responses are obtained by taking the inverse fourier transform. The users and the receiver antenna element $1\left(R x_{1}\right)$ are placed in a fixed position while the receiving antenna element $2\left(R x_{2}\right)$ is moved away from the $R x_{1}$ on the scale of symbol wavelength $\left(\lambda_{T}=2,4,8,12,16,20\right.$ and $24 \mathrm{~cm})$. At each position of $R x_{2}$ four radio channels were measured.

\section{Simulation Results}

The measured channel impulse responses were loaded into MATLAB ${ }^{\circledR}$ for simulating the receiver. Figures 5 and 6 show the plots of the filter coefficients and the squared errors when the receiver antennas are separated on $\lambda_{T}=2 \mathrm{~cm}$ and $\lambda_{T}=4 \mathrm{~cm}$ respectively. Results shows that there is decrease in the MMSE values as the spacing between the antenna elements increases in an LoS scenario. And it is clear from Figure 7 that when the receiver antennas are separated by

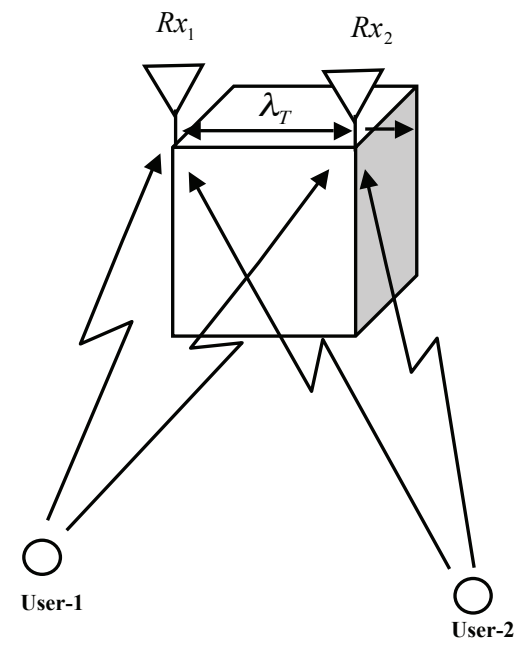

Figure 3. Measurement setup for the two-by-two system case

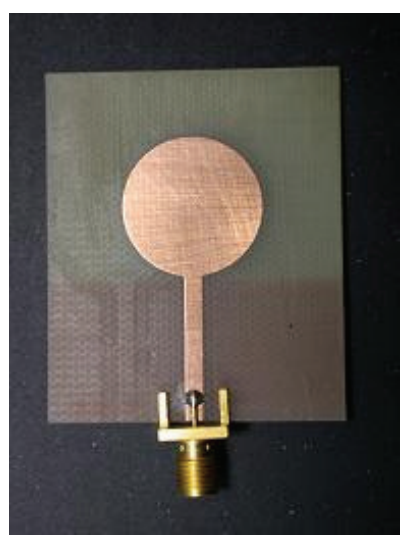

Figure 4. Picture of the fabricated antenna

a symbol wavelength $\left(\lambda_{T}=4 \mathrm{~cm}\right)$ almost all the diversity gain is achieved. Even if we increase the antenna separation to multiples of the symbol wavelength there is no significant improvement in MMSE value.

\section{Conclusions}

UWB channel measurements are obtained using a VNA in the frequency domain. The MMSE for an UWB system is simulated when the receiver antennas are separated on the scale of a symbol wavelength based on the measured UWB channel impulse response. The results show that in LoS scenarios the value of the MMSE decreases when the separation between the receiver antenna elements increases and if the separation increases to more than one symbol wavelength, there 

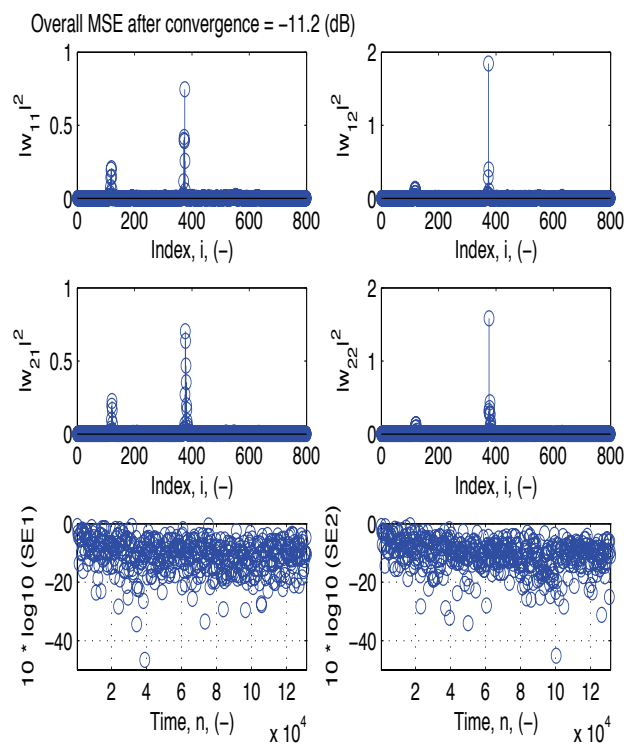

Figure 5. Filter coefficients and Squared error plots for $\lambda_{T}=2 \mathrm{~cm}$

is no significant improvement in MMSE.

\section{Acknowledgements}

This research work was funded by Atlantic Innovation Fund and by Aliant our industrial partner.

\section{References}

[1] J. H. Reed, An Introduction to Ultra Wideband Communication Systems. Upper Saddle River, New Jersey, USA: Prentice Hall PTR, first ed., 2005.

[2] H. Yanikomeroglu and E. S. Sousa, "Antenna gain against interference in CDMA macrodiversity systems," IEEE Transactions on Communications, vol. 50, pp. 1356-1371, Aug. 2002.

[3] G. Zhu, B. R. Petersen, and B. G. Colpitts, "Signalling wavelength in an antenna array for spacetime wireless over los channels," in Proceedings of the 3rd Annual Communications Networks Services and Research Conference (CNSR 2005), vol. 1, (Halifax, NS, Canada), pp. 69-73, May 16-18, 2005.

[4] M. Chamchoy, S. Promwong, P. Tangtisanon, and J.-I. Takada, "Spatial correlation properties of multiantenna UWB systems for in-home scenarios,"
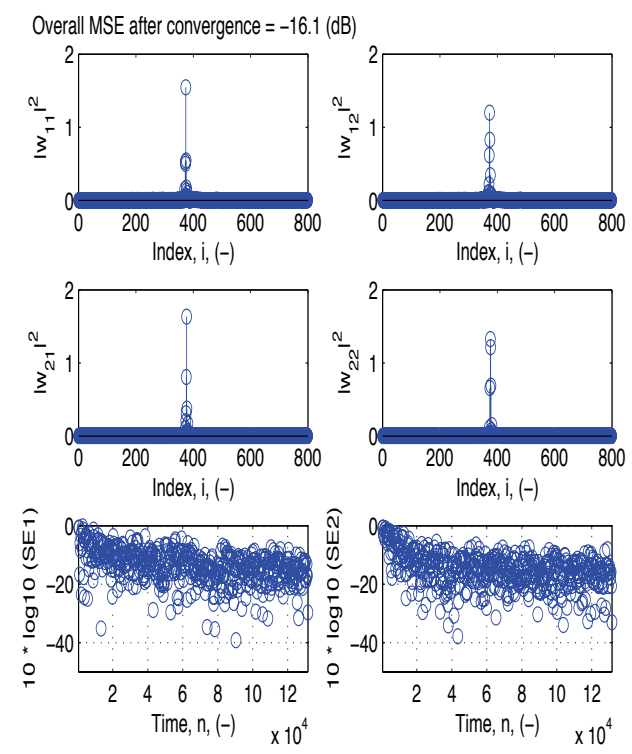

Figure 6. Filter coefficients and Squared error plots for $\lambda_{T}=4 \mathrm{~cm}$

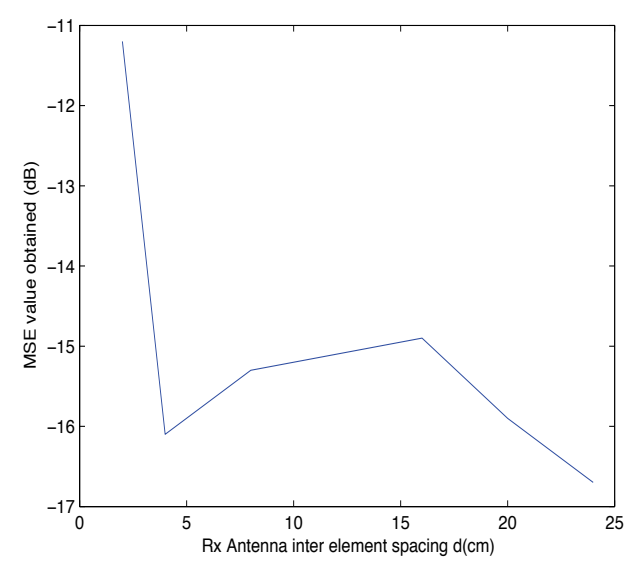

Figure 7. $M S E V s \lambda_{T}$

in IEEE International Symposium on Communications and Information Technology 2004 (ISCIT 2004), vol. 2, (Sapporo, Japan), pp. 1029-1032, Oct. 26-29 2004.

[5] G. K. Zhu, "On the signalling length in digital wireless communications," MScE Thesis, University of New Brunswick, Fredericton, Canada, 2005.

[6] N. Amitay and J. Salz, "Linear equalization theory in digital transmission over dually polarized fading 
radio channels," ATEST Tech. J., vol. 63, pp. 22152259, Dec. 1984.

[7] J. Liang, C. C. Chiau, X. Chen, and C. G. Parini, "Printed circular disc monopole antenna for ultra wideband applications," IEEE Electronics Letters, vol. 40, pp. 1246-1247, Sept. 2004.

[8] J. Powell and A. Chandrakasan, "Differential and single ended elliptical antennas for 3.1-10.6 GHz ultra wideband communication," in IEEE Antennas and Propagation Society International Symposium, vol. 3, pp. 2935-2938, Jun. 2004.

[9] T. S. Rappaport, Wireless Communications. Upper Saddle River, New Jersey, USA: Prentice Hall PTR, 1996. 